\title{
A contribuição na disseminação do conhecimento de Oftalmologia da primeira Liga Acadêmica de Belo Horizonte - um relato de experiência
}

\author{
Contribution in the dissemination of knowledge in Ophthalmology of the first \\ Academic League of Belo Horizonte - an experience report \\ Roberto Ferreira de Almeida Araújo ${ }^{1}$ (D), Bruna Stefane Silva Cotta ${ }^{2}$ (D), Leonardo Santos Resende ${ }^{1}$ (D), \\ Rodrigo Pinto Coelho Alvarenga ${ }^{3}$ (D), Larissa Lima Magalhães ${ }^{1}$ (D), Fábio Nishimura Kanadani ${ }^{4}$ (D)
}

\begin{abstract}
RESUMO
As ligas acadêmicas estão presentes no Brasil desde 1920 (Liga de Combate à Sífilis e outras Doenças Sexualmente Transmissíveis). As atividades são estruturadas a partir da tríade: ensino, pesquisa e extensão. A Liga Acadêmica de Oftalmologia (OFTALMOLIGA) da Faculdade Ciências Médicas de Minas Gerais (FCMMG), fundada em 2012, proporciona aos alunos um maior contato com a oftalmologia, que é negligenciada na maioria dos currículos obrigatórios de cursos de graduação. Objetiva-se, com este trabalho, descrever as atividades vivenciadas durante a gestão de uma liga acadêmica de oftalmologia entre os anos de 2017-2019. A OFTALMOLIGA é uma agremiação de alunos bastante ativa do ponto de vista teórico-prático-científico. Ao longo do período descrito, uma quantidade expressiva de textos científicos (Artigos) e trabalhos para eventos médicos foram produzidas. Ser membro integrante da OFTALMOLIGA é algo almejado por muitos acadêmicos da FCMMG e de outras instituições de ensino da Região Metropolitana de Belo Horizonte. Nós acreditamos que o contato dos acadêmicos nas ligas de diferentes especialidades pode ajudá-los na escolha do caminho a ser seguido em direção à especialização médica.
\end{abstract}

Palavras-chave: Educação Médica; Oftalmologia; Escolha da Profissão; Educação de Graduação em Medicina; Estudantes de Medicina; Desempenho Acadêmico.

\begin{abstract}
The academic leagues are in Brazil since 1920 (League of Combat against Syphilis and other Sexually Transmitted Diseases). The activities are based on the triad: teaching, research, and extension. The Academic League of Ophthalmology (OFTALMOLIGA) of Medical Sciences Faculty of Minas Gerais (FCMMG), founded in 2012, provides to the medical students a greater contact with the ophthalmology that is neglected in the majority of the compulsory curriculum of the Medical course. This work aims to describe the activities experienced during the management of an academic league of ophthalmology between the years 2017-2019. OFTALMOLIGA is a student group plenty active from the point of view theoretical-practical-scientific. Over the period analyzed, an expressive amount of scientific production was published in scientific Journals and medical conferences. Being a member of OFTALMOLIGA is something desired by many FCMMG's medical students and from other institutions at the Metropolitan region of Belo Horizonte. We believe that the contact with different leagues during the Medical course may help students to decide better their future professional steps.
\end{abstract}

Keywords: Medical Education; Ophthalmology; Career Choice; Medical Education Undergraduate; Students, Medical; Academic Performance.

1. Acadêmico de Medicina da Faculdade de Ciências Médicas de Minas Gerais (FCMMG), Belo Horizonte (MG), Brasil.

2. Especializanda de Oftalmologia do Instituto de Olhos Ciências Médicas, Belo Horizonte (MG), Brasil.

3. Acadêmico de Medicina da Universidade Federal de Minas Gerais (UFMG), Belo Horizonte (MG), Brasil.

4. Professor titular da cadeira de Oftalmologia (FCMMG), Pesquisador colaborador da Mayo Clinic's, Jacksonville, (FL), Estados Unidos.

$\square$ Roberto Ferreira de Almeida Araújo. Rua Gonçalves Dias, 1508, apto 701 - Lourdes. CEP 30.140-092, Belo Horizonte (MG). robertofaaraujo@gmail.com | Recebido: 15/02/2019 | Aprovado: 27/09/2019 


\section{INTRODUÇÃO}

A Faculdade de Ciências Médicas de Minas Gerais (FCMMG) fundada no ano de 1950, na cidade de Belo Horizonte, Minas Gerais, é uma instituição educacional de ensino superior privada centrada nos cursos de graduação da área de ciências da saúde, dentre eles Medicina, Fisioterapia, Enfermagem e Psicologia. Uma das atividades extracurriculares desenvolvidas dentro da instituição destaca-se a presença marcante das denominadas Ligas Acadêmicas (LAs). Atualmente, as LAs são regulamentadas por um órgão institucional denominado Coordenação de Pesquisa e Extensão (CPE) em um total de 20 ligas acadêmicas. Nesta instituição, devido às demandas crescentes pelo mercado de trabalho por um profissional que apresente elevado e sólido conhecimento teórico, e também outras habilidades como liderança, facilidade de trabalhar em equipe e bom relacionamento interpessoal em ambiente multiprofissional, as ligas acadêmicas apresentam, portanto, caráter interdisciplinar e podem ser ocupadas por estudantes de todos os cursos da área da saúde que não apenas o curso de Medicina.

Mas, afinal o quê são as ligas acadêmicas? A história nos revela que a experiência das ligas como um fenômeno de articulação e mobilização de indivíduos ou grupos em torno de uma necessidade ou carência remonta à Antiguidade, haja vista as ligas de Delos e do Peloponeso ${ }^{1}$. Entretanto, versões mais recentes deste tipo de agremiação podem ser observadas a partir das ligas esportivas que articulam questões e solucionam demandas relacionadas ao futebol e também das ligas camponesas em prol da reforma agrária ${ }^{1}$. Em dicionários de língua portuguesa, a palavra liga se associa à aliança, união, pacto ${ }^{2}$. De modo análogo, a físico-química versa que uma liga é produto de caráter metálico resultante da fusão de um ou de vários elementos a um metal com a finalidade de formar soluções sólidas. Tal característica deve ou pode ter norteado a denominação original da união de acadêmicos com objetivo extracurricular comum. Contudo, não há ainda um conceito bem definido do que sejam as LAs.

Revisitando a História da Medicina no Brasil, sabe-se que a primeira Liga Acadêmica foi instituída no ano de 1920 na Universidade de São Paulo (USP) com a Liga de Combate à Sífilis e outras doenças sexualmente transmissíveis. Tal agremiação de alunos objetivava que estudantes pudessem praticar os conhecimentos obtidos no ambiente universitário em prol da comunidade, o que existe até a atualidade ${ }^{3-21}$. Entretanto, o grande "boom" das LAs no Brasil ocorreu em um contexto de tensão político-social, durante o período de ditadura militar. Nesse contexto, as LAs funcionaram como instrumento de questionamento e resistência no ambiente universitário, uma vez que realizavam críticas ao modelo de ensino vigente bem como destinavam e aplicavam os avanços técnico-científicos à população que se encontrava alheia as novas invenções ${ }^{10-18}$.

Durante o período de redemocratização do país, na década de 1990, na qual houve uma ampla e geral reforma nos currículos acadêmicos dos cursos de Medicina, as ligas acadêmicas puderam se expandir e fortalecer ainda mais. E, novamente, a partir do ano de 2013, com a última reforma curricular nos cursos de Medicina no Brasil é possível observar um novo pico de crescimento das LAs no ambiente universitário brasileiro ${ }^{10-18}$.

A complexidade ao redor da criação das LAs no Brasil é tamanha que durante o VIII Congresso Brasileiro de Clínica Médica realizado em Gramado, Rio Grande do Sul no ano 2006, foi criada a Associação Brasileira de Ligas Acadêmicas de Medicina (ABLAM) ${ }^{2-4,7,8,14,20}$. Posteriormente, em Assembleia Geral, foram estabelecidas Diretrizes Nacionais para regulamentar a elaboração de documentos (ata, estatutos, normas, etc.) necessários para a criação de uma Liga Acadêmica de Medicina no Brasil ${ }^{8}$.

Em estudo realizado na Faculdade de Medicina de Ribeirão Preto ${ }^{14,22}$, investigaram-se as principais atividades extracurriculares frequentadas pelos alunos do $1^{\circ}$ ao $4^{\circ}$ ano. Vieira et al. ${ }^{22}$ encontraram que em 2002, 72,6\% dos alunos frequentavam alguma liga. Tal número se revelou maior que os $58,5 \%$ que frequentavam ligas apenas três anos antes. No estudo, este crescimento foi atribuído ao próprio crescimento do número de ligas ${ }^{22}$. As ligas possuem participação opcional, sendo ambientes teoricamente livres de formalidades acadêmicas, com um sistema de autogestão do aprendizado e, dessa forma, proporcionam uma suavização do ambiente hierárquico clássico da relação professor-aluno. Logo, entende-se que 
- aprendizado dentro desses espaços torna-se mais prazeroso para o aluno ${ }^{14,22}$. Outros estudos avaliaram o impacto da criação de ligas acadêmicas no ensino de especialidades, como o estudo de De Deus Vieira et al. ${ }^{23}$, sobre a Liga de Ortopedia e Traumatologia em Rondônia. Neste estudo ponderou-se vários aspectos relacionados à criação de uma liga acadêmica tais como, a aquisição de conhecimento por meio de atividades práticas e teóricas proporcionadas pela Liga de Ortopedia. O desenvolvimento de habilidades de comunicação também foi algo de extrema relevância e tal habilidade foi possível de ser desenvolvida uma vez que os ligantes e diretores se depararam com problemas diversos. Tais problemas exigiam dos membros ligantes e diretores capacidade de diálogo com outros acadêmicos, médicos ortopedistas e outros profissionais da área da saúde ${ }^{23}$.

$E m$ relação às principais críticas às LAs, Santana et al. ${ }^{2}$ afirmam que as LAs podem ser um espaço de especialização precoce dos estudantes, sendo estimuladas pelo desenvolvimento de atividades relacionadas a somente uma área específica. Assim, os estudantes acabam por desprezar (intencionalmente ou não) as atividades próprias do curso médico para se dedicarem mais às atividades das LAs ${ }^{2,16}$.

Apesar do longo período de existência das ligas, o que se observa são inúmeras definições e funções relacionadas ao seu funcionamento. As LAs, portanto, são definidas de modo genérico como agremiações de alunos de diferentes anos da graduação médica que buscam aprimorar seus conhecimentos, sob a supervisão de um professor (orientador), guiados pelos princípios do tripé universitário de ensino, pesquisa e extensão (Figura 1). As atividades de ensino incluem aulas teóricas, discussão de casos clínicos, seminários, minicursos, simpósios, congressos e atividades práticas, como o acompanhamento de ambulatórios e outros serviços ${ }^{5}$. Já as atividades de promoção à saúde (extensão), geralmente negligenciadas nos currículos das Faculdades de Medicina, são uma das mais importantes atividades promovidas pelas LAs. Nesse contexto, podem ser realizadas campanhas de saúde em colaboração com organizações não governamentais e centros comunitários. E, tal convivência com a prática da especialidade pode contribuir na escolha da futura especialidade pelos alunos. A extensão promove a aproximação entre os profissionais em formação e a realidade em que estão inseridos, a partir do reconhecimento das necessidades populacionais, estabelecendo a comunicação entre a universidade e a sociedade de modo a preparar os alunos para atuar em um modelo de atenção à saúde que reconheça as necessidades da população ${ }^{2,4,10,19}$.

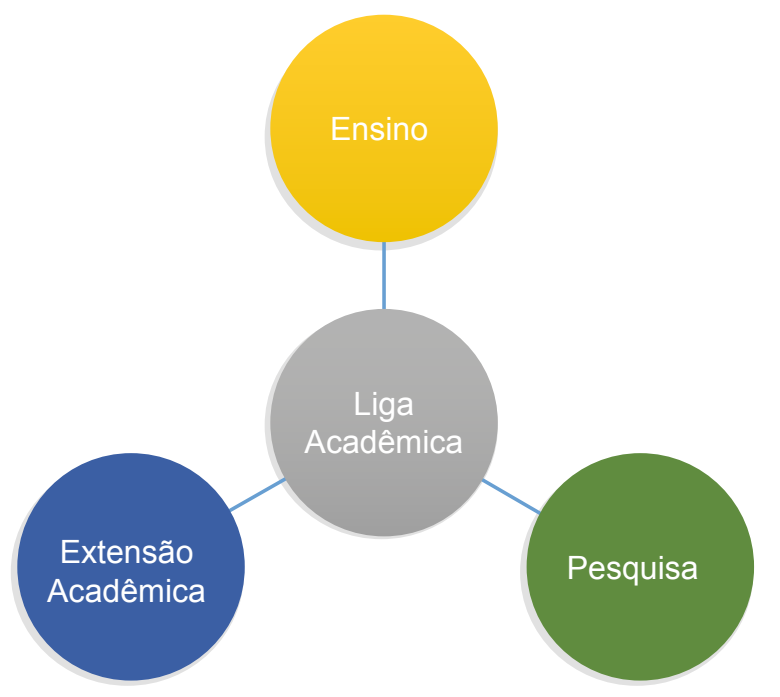

Figura 1: Tripé da Liga Acadêmica: Ensino; Pesquisa e Extensão Acadêmica.

A fomentação do conhecimento científico, relativa ao pilar de pesquisa integrante do tripé das LAs, pode gerar impactos significativos na atuação profissional do acadêmico e resultar em estudantes mais críticos, com maior conhecimento da escrita, condução de projetos científicos e questionamento dos estudos existentes. Em linhas gerais, o que pode ser definido é que a partir do momento de ingresso em uma liga, o discente amplia o seu senso crítico e raciocínio científico ${ }^{24}$, o que é relacionado a vários aspectos benéficos no contexto de sua formação. Assim, tais estratégias permitem que o educando se torne o centro de sua própria educação ao ocupar o papel de protagonista do processo de ensino-aprendizagem, evidenciando uma prática pedagógica que instigue sua criatividade, criticismo, autonomia e liberdade ${ }^{2}$.

A literatura disponível acerca das LAs é escassa, e exclusivamente nacional, sem paralelos em referências internacionais, uma vez que é um fenômeno muito particular da realidade do ambiente universitário brasileiro ${ }^{14}$. Nota-se, entre- 
tanto, um aumento gradual do debate a respeito de LAs e de seu impacto na formação do médico. Além das publicações, muitos eventos de educação médica têm reservado espaço para discutir o tema ${ }^{14}$. O presente trabalho tem como objetivo relatar a experiência de uma Liga Acadêmica de Oftalmologia e discutir o papel das ligas acadêmicas a partir da experiência com as atividades da Liga Acadêmica de Oftalmologia (OFTALMOLIGA) realizadas durante o triênio 2017-2019 no desenvolvimento de atividades extracurriculares por discentes e docentes do Curso Médico da Faculdade de Ciências Médicas de Minas Gerais bem como sua importância para a formação médica.

\section{A estruturação da oftalmoliga}

A Liga Acadêmica de Oftalmologia (OFTALMOLIGA) é vinculada à CPE da FCMMG e existe desde o ano de 2012, tendo sido idealizada por um grupo de alunos do curso de Medicina da FCMMG. A OFTALMOLIGA é a liga mais antiga da especialidade de oftalmologia em Belo Horizonte e é vinculada à Associação Brasileira de Ligas Acadêmicas de Oftalmologia (ABLAO) ${ }^{25}$. Além disso, a OFTALMOLIGA, é uma liga que agrega acadêmicos de todos os cursos da área da saúde devido à complexidade das doenças que acometem o olho e priorizando uma visão global e não segmentada do indivíduo.

\section{Processo seletivo e estruturação teórica}

Acadêmicos de todos os cursos da saúde da FCMMG e de outras instituições da região Metropolitana de Belo Horizonte podem ingressar na Liga Acadêmica de Oftalmologia (OFTALMOLIGA) através do processo seletivo anual, realizado conforme o Estatuto da liga e edital próprio para cada processo. No ano de 2017 , o processo seletivo foi realizado no dia 23 de maio e no ano seguinte, no dia 10 de abril, ambos nas dependências da FCMMG. Essas seleções foram realizadas por meio de um teste objetivo, contendo 30 questões diversas abordando temas gerais da especialidade. Os resultados das provas foram divulgados no dia seguinte, sendo que respectivamente 16 e 21 novos acadêmicos foram selecionados para integrar o corpo discente da liga junto a respectivamente oito e nove membros diretores da gestão anterior. As atividades de ensino em ambos os anos foram compostas por aulas teóricas/práticas com frequências quinzenais realizadas nas dependências da FCMMG e ministradas por médicos oftalmologistas e residentes do Instituto de Olhos Ciências Médicas, este vinculado ao Hospital Universitário Ciências Médicas. Os temas abordados no período de 2017-2018 e 2018-2019 foram: anatomia ocular; anatomia da córnea e filme lacrimal; embriologia ocular; semiologia oftalmológica; oftalmopediatria; ametropias; olho vermelho; farmacologia ocular; catarata - fisiopatologia, diagnóstico e terapêutica; glaucoma - fisiopatologia, diagnóstico e terapêutica; retinopatia diabética; retinopatia hipertensiva; doença macular relacionada à idade; celulite periorbitária e seus diagnósticos diferenciais; traumas e urgências oftalmológicas; manifestações oftalmológicas de doenças sistêmicas; transplante de córnea; por que oftalmologia? A metodologia utilizada consistiu na apresentação visual de pontos importantes para a compreensão teórica dos temas bem como associação com possíveis condutas na prática médica.

\section{Atividades práticas}

No ano de 2017, a diretoria da liga instituiu uma atividade prática inovadora entre as ligas de Oftalmologia de Belo Horizonte: a aula prática de dissecação do globo ocular (Figura 2), com o professor e médico oftalmologista Dr. Fábio Nishimura Kanadani, orientador da OFTALMOLIGA. Essa aula foi muito bem avaliada entre os acadêmicos membros da liga, e por ter sido ministrada no início do semestre, trouxe grande interesse e motivação no estudo da Oftalmologia para os ingressantes. 


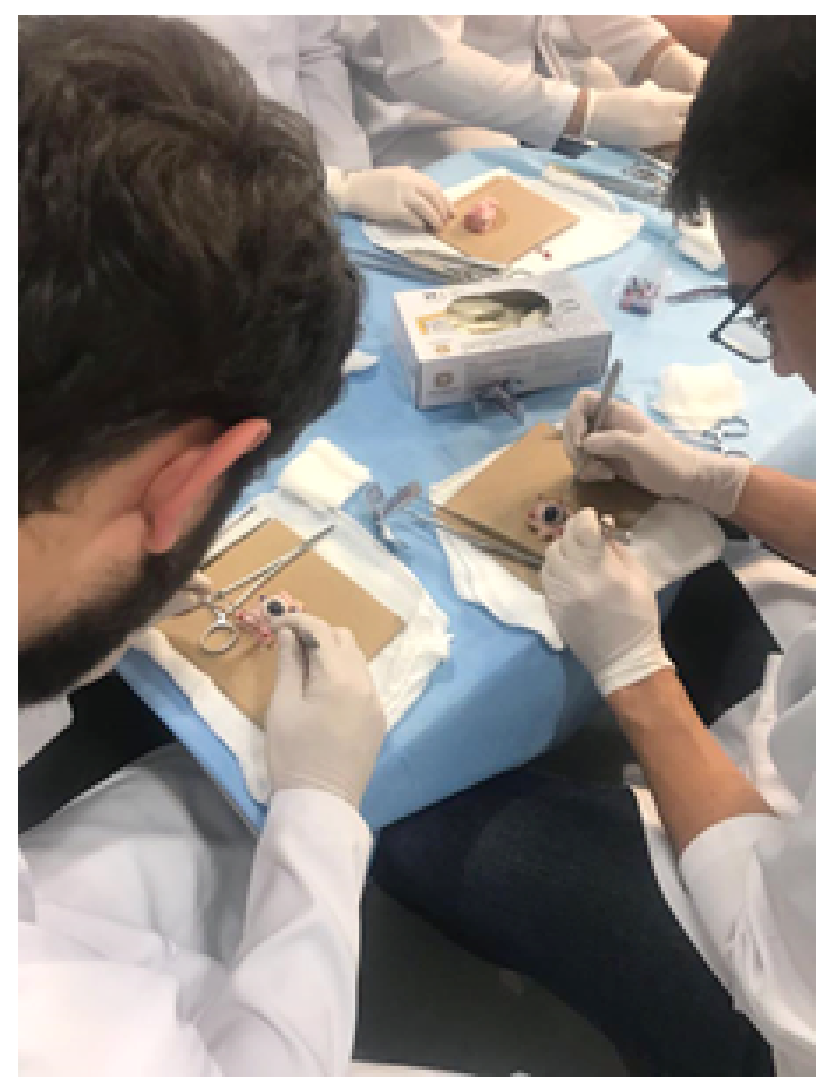

Figura 2: Aula de dissecação do globo ocular em olho de Sus scrofa domesticus (porco doméstico).

As demais atividades práticas da OFTALMOLIGA aconteceram nas dependências do Instituto de Olhos Ciências Médicas. Os acadêmicos foram divididos em grupos de 10 alunos para melhor execução das atividades práticas programadas por semestre. No primeiro semestre do ano, 10 alunos frequentaram individualmente 0 serviço de oftalmologia com uma frequência de 6 horas semanais, sempre sob a supervisão de residentes e preceptores do serviço. As atividades práticas ambulatoriais envolveram a participação dos acadêmicos em todos os ambulatórios de especialidade e também no bloco cirúrgico da instituição, possibilitando o contato precoce dos ligantes com as várias subespecialidades da oftalmologia, como: córnea; plástica ocular; glaucoma; retina; refração; lentes de contato; neuro-oftalmologia e estrabismo; catarata e uveítes.

\section{Organização de eventos científicos}

Para divulgar o conhecimento em Oftalmologia no meio acadêmico, no ano de 2017 também foi organizado, através de agremiação entre a diretoria da OFTALMOLIGA e alguns membros ligantes, o I Congresso Acadêmico de Oftalmologia (I CAO-MG). Foi o primeiro evento científico na área de Oftalmologia organizado totalmente por acadêmicos de Medicina. O I CAO-MG ocorreu no dia 18 de novembro de 2017, na Associação Médica de Minas Gerais (AMMG), valendo certificação de 15 horas ( 1 crédito) para seus participantes. O congresso contou com a participação de oftalmologistas renomados como palestrantes, somando um total de 11 palestras de temas da oftalmologia, além das apresentações de empresas patrocinadoras. O evento foi um sucesso, assim, nos dias 14 e 15 setembro de 2018 aconteceu a sua segunda edição - II CAO-MG. Os dois primeiros eventos contaram com a presença de mais de 180 participantes inscritos, dentre eles, acadêmicos e alguns residentes/especializandos em oftalmologia. O II Congresso Acadêmico de Oftalmologia de Minas Gerais (Figura 3) proporcionou aos seus participantes a possibilidade de publicação de trabalhos na forma de artigo completo (os três melhores trabalhos na categoria pôster) ou resumo (um total de 35 trabalhos) na Revista Médica de Minas Gerais (RMMG). A RMMG apresenta indexação nas bases de dados Literatura Latino-Americana e do Caribe em Ciências da Saúde (LILACS); Índice de Revistas Latinoamericanas (PERIODICA) e Sistema Regional de Información em Línea para Revistas Científicas da América Latina, El Caribe y Portugal (LATINDEX). Essa oportunidade de publicação científica em revista indexada foi um grande atrativo para que os estudantes de todas as faculdades da região metropolitana participassem do evento, colaborando para este ser um novo sucesso.

O III CAO-MG, a ser realizado neste ano de 2019 já conta com quase 300 participantes. Isso reforça a existência de uma carência de informação sobre a especialidade de Oftalmologia que pode ser suprida, ao menos parcialmente, pela OFTALMOLIGA. 


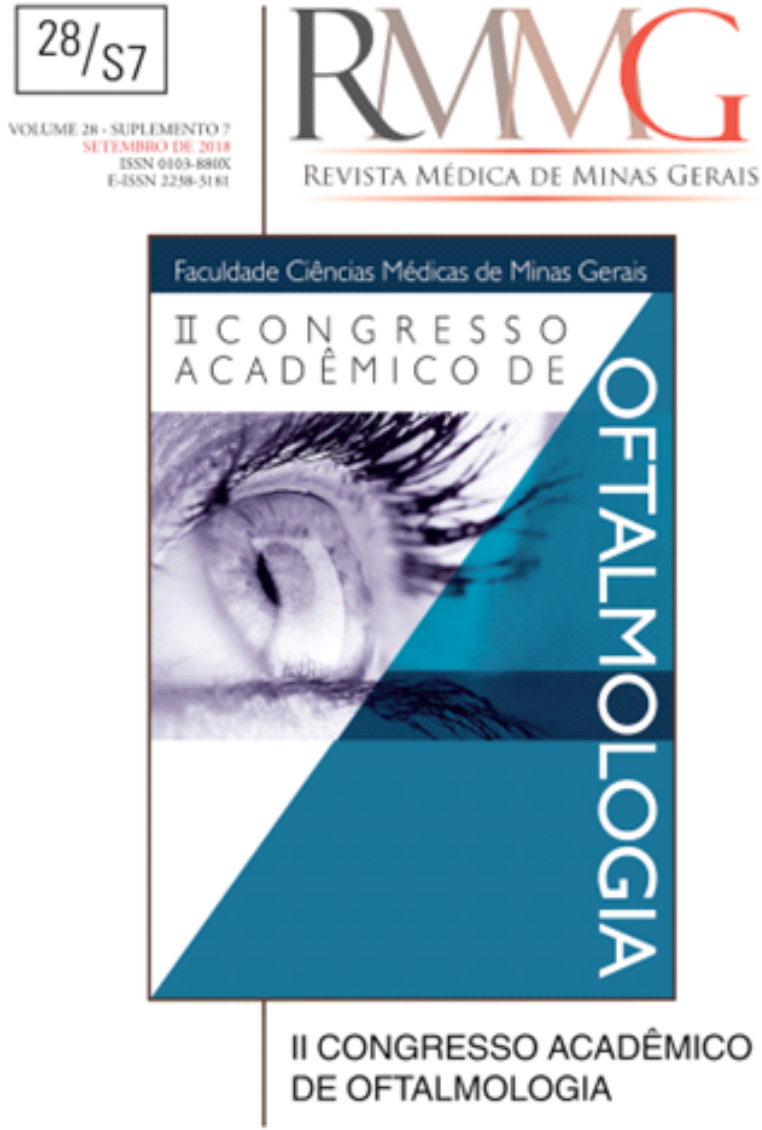

Figura 3: Capa da edição de suplemento da II Congresso Acadêmico de Oftalmologia na Revista Médica de Minas Gerias.

\section{Atividades de extensão e convívio na comunidade}

Dentre as atividades de extensão realizadas pela OFTALMOLIGA, tem-se a realização de campanhas de saúde com pacientes do ambulatório Ciências Médicas e também do Instituto de Olhos de Minas Gerais, a partir da abordagem das principais afecções que acometem o olho e fornecendo informações a respeito de prevenção dessas patologias. No período de março de 2017 até março de 2019, destaca-se a realização de uma atividade em parceria com a Liga Acadêmica de Endocrinologia e Oftalmologia (LAEM) da FCMMG em comemoração ao novembro Azul da Diabetes. Tal atividade foi realizada em inúmeros dias em diversos locais como o ambulatório Ciências Médicas, Instituto de Olhos Ciências Médicas e também em locais públicos como o Mercado Central de Belo Horizonte, e em uma dessas datas houve uma cobertura da imprensa local filiada à rede Globo, dando destaque à iniciativa realizada pelos estudantes (Figura 4). Tal parceria entre ligas acadêmicas foi uma grande oportunidade para os acadêmicos de ambas integrarem os seus conhecimentos adquiridos em sala de aula em um cenário prático, inseridos na comunidade, tendo assim a possibilidade de exercitar as habilidades de relacionamento interpessoal, postura e ética na profissão.

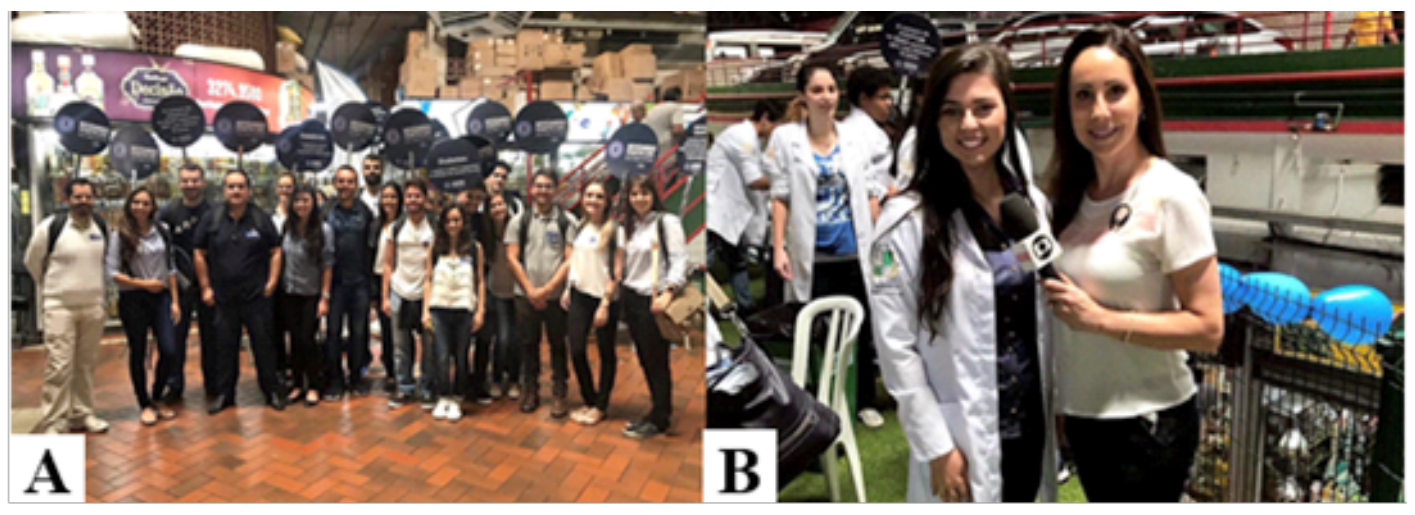

Figura 4: Ação de extensão no Mercado Central de Belo Horizonte. (A) Grupo de alunos no Mercado Central integrantes da OFTALMOLIGA e da LAEM. (B) Diretora da OFTALMOLIGA em entrevista à filial da rede Globo de Belo Horizonte Minas Gerais.

\section{Atividades de pesquisa e iniciação científica}

Por último, em relação às atividades de pesquisa no período abordado houve três projetos de iniciação científica financiados por órgão de fomento à pesquisa, Fundação de Amparo à Pesquisa do Estado de Minas Gerais (FAPEMIG), realizados por três alunos vinculados à liga. Os projetos executados nos anos 2017-2018 foram intitulados: 
"Avaliação de pacientes em terapia intravítrea com antiangiogênicos no Instituto de Olhos Ciências Médicas" e "Avaliação de fatores prognósticos a curto prazo com o uso de ranibizumabe em pacientes com edema macular diabético"; e o projeto executado nos anos 2018-2019 foi intitulado: "Elaboração de um protocolo para determinar o intervalo pós-morte por meio da pressão intraocular". Além disso, anualmente na cidade de Belo Horizonte ocorre um evento médico regional na área de oftalmologia - o Congresso do Hospital São Geraldo. Durante os anos de 2017 e 2018, sete trabalhos foram apresentados por acadêmicos vinculados à OFTALMOLIGA, a saber: "Oclusão de Artéria Central da Retina: um relato de caso"; "Glaucoma Primário de Ângulo Aberto e a Síndrome de Fong"; "Importância Do Diagnóstico Diferencial da Síndrome de Posner-Schlossman e as Uveítes Glaucomatosas"; "Manifestações Oftalmológicas na Malformação de Chiari Tipo I". Foram publicados em revistas da área médica três artigos originais entre os anos de 2017 e 2019, o que totalizou 10 trabalhos no período dessa gestão (Tabela 1 ).

\section{Tabela 1}

Relação de Trabalhos publicados no triênio de 2017-2019 pela Liga Acadêmica de Oftalmologia

\begin{tabular}{|c|c|c|c|c|c|}
\hline Número & Título do Trabalho & $\begin{array}{c}\text { Categoria } \\
\text { (Evento Médico/Artigo) }\end{array}$ & $\begin{array}{l}\text { Nota } \\
\text { Qualis }\end{array}$ & $\begin{array}{l}\text { Fator de } \\
\text { Impacto }\end{array}$ & $\begin{array}{c}\text { Ano de } \\
\text { Publicação }\end{array}$ \\
\hline 1 & $\begin{array}{l}\text { Avaliação de fatores prognósticos a curto } \\
\text { prazo com o uso de ranibizumabe em } \\
\text { pacientes com edema macular diabético }{ }^{26}\end{array}$ & RBO (ARTIGO) & B3 & $\begin{array}{l}\text { SJR 2017: } \\
\quad 0.15\end{array}$ & 2018 \\
\hline 2 & $\begin{array}{l}\text { Perfil de pacientes em terapia intravítrea } \\
\text { com antiangiogênicos no Instituto de Olhos } \\
\text { Ciências Médicas }{ }^{27}\end{array}$ & RMMG (ARTIGO) & $\mathrm{C}$ & NA & 2018 \\
\hline 3 & $\begin{array}{l}\text { Síndrome de Axenfeld-Rieger: a importân- } \\
\text { cia do diagnóstico precoce e da avaliação } \\
\text { oftalmológica }{ }^{28}\end{array}$ & RMMG (ARTIGO) & $\mathrm{C}$ & NA & 2018 \\
\hline 4 & Glaucoma de pressão normal & $\begin{array}{l}\text { APRESENTAÇÃO ORAL } \\
\text { (XXXVIII CHSG) }\end{array}$ & NA & NA & 2018 \\
\hline 5 & $\begin{array}{l}\text { Baixa de visão, cefaleia e zumbido: } \\
\text { O que fazer? }\end{array}$ & $\begin{array}{l}\text { APRESENTAÇÃO ORAL } \\
\text { (XXXVIII CHSG) }\end{array}$ & NA & NA & 2018 \\
\hline 5 & $\begin{array}{l}\text { A Importância do Diagnóstico Diferencial } \\
\text { do Hemangioma Cavernoso Retiniano }\end{array}$ & $\begin{array}{c}\text { PÔSTER } \\
(X X X V I I I \text { CHSG })\end{array}$ & NA & NA & 2018 \\
\hline 6 & $\begin{array}{l}\text { Diagnóstico Tardio de Síndrome da } \\
\text { Rubéola Congênita: relato de caso }\end{array}$ & $\begin{array}{c}\text { PÔSTER } \\
(\mathrm{XXXVIII} \mathrm{CHSG)} \\
\end{array}$ & NA & NA & 2018 \\
\hline 7 & $\begin{array}{l}\text { Oclusão de Artéria Central da Retina: } \\
\text { um relato de caso }\end{array}$ & $\begin{array}{c}\text { POSTER } \\
(\text { XXXVII CHSG) }\end{array}$ & NA & NA & 2017 \\
\hline 8 & $\begin{array}{l}\text { Glaucoma Primário de Ângulo Aberto e a } \\
\text { Síndrome de Fong }\end{array}$ & $\begin{array}{l}\text { APRESENTAÇÃO ORAL } \\
\text { (XXXVII CHSG) }\end{array}$ & NA & NA & 2017 \\
\hline 9 & $\begin{array}{l}\text { Importância do Diagnóstico Diferencial da } \\
\text { Síndrome de Posner-Schlossman e as } \\
\text { Uveítes Glaucomatosas }\end{array}$ & $\begin{array}{c}\text { POSTER } \\
(\mathrm{XXXVII} \mathrm{CHSG)}\end{array}$ & NA & NA & 2017 \\
\hline 10 & $\begin{array}{l}\text { Manifestações Oftalmológicas na } \\
\text { Malformação de Chiari Tipo I }\end{array}$ & $\begin{array}{l}\text { APRESENTAÇÃO ORAL } \\
\text { (XXXVII CHSG) }\end{array}$ & NA & NA & 2017 \\
\hline
\end{tabular}

Nota: RBO= Revista Brasileira de Oftalmologia. RMMG= Revista Médica de Minas Gerais. NA= Não se aplica. $\mathrm{CHSG}=\mathrm{Congresso}$ do Hospital São Geraldo.

\section{Importância das ligas na formação médica e situação atual}

A contribuição na formação médica pelas denominadas ligas acadêmicas já é algo bastante explorado pela literatura médica existente, e o que se observa são inúmeros benefícios ${ }^{30,31,32}$.
Um desses benefícios é a bagagem de aulas teóricas, que são de fundamental importância para o aprendizado dos alunos. Por tratarem de temas não abordados no currículo tradicional da maioria das faculdades de medicina, tais aulas permitem um melhor preparo dos acadêmicos ligantes frente às queixas oftalmológicas a que 
serão submetidos em suas práticas clínicas como médicos generalistas. As aulas práticas, como a de dissecação do globo ocular, permitem que os acadêmicos se familiarizem melhor com as estruturas do globo ocular e ferramentas de exames próprias do oftalmologista. Além disso, nesse cenário o aluno assume um papel ativo na aquisição e fixação do conhecimento ${ }^{29,30}$.

Em relação às atividades relacionados ao Congresso Acadêmico é sabido que após a graduação médica, muitos profissionais utilizam como forma de se atualizarem e de aprimorarem os seus conhecimentos os eventos relacionados à sua área de atuação: cursos, congressos, jornadas e simpósios. Introduzir esse espaço de discussão e de aprendizado de maneira precoce na vida do acadêmico de medicina é benéfico, uma vez que tais ações incentivam o estudante a sempre buscar por mais conhecimento. Além disso, a possibilidade de poder apresentar trabalhos publicáveis torna o acadêmico mais interessado em desenvolver atividades relacionadas ao ensino e à pesquisa.

$\mathrm{Na}$ extensão acadêmica, o conhecimento adquirido nas Instituições de Ensino Superior (IES) é compartilhado com a sociedade de maneira a promover a prevenção de agravos de saúde e informar a população sobre tópicos importantes relacionados às principais patologias que acometem o sistema visual. Os estudantes vinculados às ligas acadêmicas, ao desenvolver tais atividades, realizam o árduo exercício de popularizar o conhecimento adquirido dentro da academia em prol do bem-estar da população ${ }^{4}$.

Por fim, em relação às atividades de pesquisa e, levando em conta a elevada produção científica realizada pelos acadêmicos membros da OFTALMOLIGA, observa-se um aumento do interesse dos acadêmicos em eventos científicos da área médica, dando expressividade à presença dos estudantes nesses ambientes, outrora tão fechados a eles. Dessa forma, as LAs ensinam a importância da pesquisa, atividade indispensável na busca por melhores resultados terapêuticos e, consequentemente, condutas mais acertadas para os pacientes. Tal atividade deve fazer parte do cotidiano médico e, portanto, a OFTALMOLIGA insere essa prática precocemente aos estudantes.

\section{CONCLUSÃO}

As ligas acadêmicas têm se firmado como uma das atividades extracurriculares mais dinâmicas da formação médica atual, e nesse contexto, as atividades da OFTALMOLIGA, extremamente diversas e integradas no tripé de ensino-pesquisa-extensão são um exemplo exitoso desse fenômeno na educação médica na cidade de Belo Horizonte. Concluímos que a OFTALMOLIGA, bem como as demais LAs, são espaços importantes para que os estudantes se preparem para a prática médica, conhecendo as diferentes áreas da Medicina e dessa forma auxiliando-os na opção da futura especialidade.

Nesse sentido, a OFTALMOLIGA vem se tornando uma referência municipal e estadual para acadêmicos que têm interesse na área da oftalmologia. O relato de tal experiência positiva na FCMMG nos leva a crer que iniciativas como essa devam ser não somente divulgadas como reproduzidas em outras instituições de ensino superior (IES).

\section{Agradecimentos}

"Gostaríamos de agradecer a toda a equipe fundadora da OFTALMOLIGA do ano de 2012, em especial, a Dra. Rafaela de Morais Miranda e a Dra. Maisa Dias Pinto Coelho, pois sem vocês nada disso hoje seria possível."

\section{REFERÊNCIAS}

1. Silva SA, Flores O. Ligas Acadêmicas no Processo de Formação dos Estudantes. Rev Bras Educ Med. 2015;39(3):410-7.

2. Santana ACDA. Ligas acadêmicas estudantis. O médico e a realidade. Medicina (Ribeirao Preto, Online). 2012;45(1):96-8.

3. Cavalcante ASP, Vasconcelos MIO, Lira GV, Henriques RLM, Albuquerque INMA, Maciel GP, et al. The Academic Leagues in the Health Area: Knowledge Gaps from the Brazilian Scientific Production. Rev Bras Educ Med. 2018;42(1):199-206.

4. Melo Neto AP, Martins AF, Alves PVB, Carvalho VR, Melo PGA, Pinheiro VGF. Liga acadêmica do pulmão: extensão universitária como parte da formação médica. Sanare, Revista de Políticas Públicas. 2015;14(2)135-40.

5. Barbieri LG, Mendonça RR, Guimarães DF, Barbieri DGFV. Liga acadêmica de fisioterapia em ortopedia e trauma- 
tologia no esporte--lorte: um relato de experiência. Rev Ciênc Ext. 2015;11(1)161-70.

6. Holanda VN, Sousa AK, Vandesmet VCS, Torres CMG, Milfonte WG, Bezerra AS, et al. Liga acadêmica de bioquímica clínica: experiência de implantação e participação na primeira liga acadêmica numa instituição de ensino superior de Juazeiro do Norte-Ceará. Revista de Ensino de Bioquímica. 2015;13(3):87-99.

7. Silva JHS, Chiochettal LG, Oliveira LFT, Sousa VO, et al. Implantação de uma Liga Acadêmica de Anatomia: Desafios e Conquistas. Rev Bras Educ Med. 2015;39(2):310-5.

8. Silva AS, Flores O. Ligas Acadêmicas no Processo de Formação dos Estudantes. Rev Bras Educ Med. 2015;39(3):410-7.

9. Queiroz SJ, Andrade M, Casas ALF, Santos GMDS, Chiochetti NB. Ligas Acadêmicas e Currículo Médico: relações e relato de experiência. Fragmentos de Cultura. $2015 ; 25(1): 47-55$.

10. Montiel EMS, Stumpf MAM, Okamoto JM, Rodrigues MRS, Kopruszynski CP, Schrut GCA. Relatos das ações extensionistas da liga acadêmica do trato gastrointestinal e doenças metabólicas. Revista Conexão UEPG. $2016 ; 12(3): 436-46$.

11. Andreola GM, Brenner FAM. O papel das Ligas Acadêmicas de Dermatologia na formação acadêmica: adaptando o ensino da Dermatologia aos tempos atuais. Rev Med UFPR. 2016;3(2):77-9.

12. Van Vaisberg V, Cotrim FP, Ghaffar AS, Hara LAA, Monteiro RF, Tannuri U, et al. Operation and characteristics of the Academic League of Pediatric Surgery in quaternary Center. Revista de Medicina. 2017;96(4)241-4.

13. Rodrigues MS, Galvão IM. Estudantes de medicina dos três primeiros anos são os principais ingressantes na Liga Acadêmica de Medicina de Urgência e Emergência. Revista de Medicina. 2017;96(3):136-9.

14. Goergen DI. Ligas acadêmicas: uma revisão de várias experiências. Arquivos Catarinenses de Medicina. 2017;46(3):183-93.

15. Costa BEP, Hentschke MR, Silva ACC, Barros A, Salerno M, Poli-de-Figueiredo $C E$, et al. Reflexões sobre a importância do currículo informal do estudante de medicina. Scientia Medica (Porto Alegre). 2012;22(3):162-8.

16. Botelho NM, FERREIRA IG, Souza LEA. Ligas acadêmicas de medicina: artigo de revisão. Rev Para Med. $2013 ; 27(4): 85-8$

17. Hamamoto Filho PT. Ligas acadêmicas: motivações e críticas a propósito de um repensar necessário. Rev Bras Educ Med. 2011;35(4):535-43.

18. Soares LR, Freitas-Junior R, Ribeiro LZ, Rahal RMS. Iniciação científica na graduação: experiência da Liga da Mama da Universidade Federal de Goiás. Rev Bras Mastologia. 2017;27(1).

19. Ferreira IG, Souza L, Botelho NM. Ligas Acadêmicas de Medicina: perfil e contribuições para o ensino médio. Rev Soc Bras Clín Med. 2016;14(4):239-44.
20. Mendes WO, Pereira MC, Freitas JC, Castro Junior FM. Liga de cirurgia de cabeça e pescoço da Universidade Federal do Ceará: 6 anos de ensino, pesquisa e extensão. Rev Bras Cir Cabeça Pescoço. 2014;43(3):132-6.

21. Fernandes FG, Hortêncio LOS, Unterpertinger FV, Waisberg DR, Pêgo-Fernandes PM, JATENE FB. Cardiothoracic Surgery League from University of São Paulo Medical School: twelve years in medical education experience. Braz J Cardiovas Surg. 2010;25(4):552-8.

22. Vieira EM, Barbieri CLA, Vilela DB, Ianhez Junior $E$, Tomé FS, Woida FM, et al. O que eles fazem depois da aula? As atividades extracurriculares dos alunos de ciências médicas da FMRP-USP. Medicina (Ribeirao Preto, Online). 2004;37(1/2):84-90.

23. Vieira GD, Quintana FT, Mendonça GM, Pinto ICCS, Bezerra ICA, Braga JOS, et al. Contribuição para o ensino de Ortopedia da primeira liga da especialidade em Rondônia. Medicina (Ribeirão Preto, Online). 2014;47(2):201-7.

24. Melo AMD, Silva Filho MS, Lima RBN, Medeiros BF, Aguilera KC, Alencar AMC, et al. A importância da liga acadêmica de oncologia na formação profissional e promoção da saúde: um relato de experiência. Id on Line Rev Mult Psic. 2018;12(40).

25. Kato JM, Albuquerque GP, YEH CO, Resende MF, Barros MF. Associação Brasileira de Ligas Acadêmicas de Oftalmologia: perspectivas e desafios. Revista de Medicina. 2015;94(1):48-9.

26. Kanadani TCM, Cotta BSS, Souza ACCR, Costa APO, Takahashi DM. Evaluation of short-term prognostic factors of ranibizumab in patients with dabetic macular edema. Revista Brasileira de Oftalmologia. 2018;77(3):137-41.

27. Resende LS, Pinto ICT, Magalhaes LL, Tom Back MIPSDS, Mares VSL, Araújo RFA, et al. Perfil de pacientes em terapia intravítrea com antiangiogênicos no instituto de olhos ciências médicas. Rev Med Minas Gerais. 2018;28(Supl.7):10-6.

28. Beneti GC, Barbosa JC, Faria NM, Francisco FCO, Nogueira LR, Magalhaes LL, et al. Síndrome de Axenfeld-Rieger: a importância do diagnóstico precoce e da avaliação oftalmológica. Rev Med Minas Gerais. 2018;28(Supl.7):21-5.

29. Kara José AC, Passos LB, Kara José FC, Kara José N. Ensino extracurricular em Oftalmologia: grupos de estudos/ligas de alunos de graduação. Rev Bras Educ Med. 2007;31(2):166-72.

30. Bastos MLS, Trajman A, Teixeira EG, Selig L, Belo MTCT. O papel das ligas acadêmicas na formação profissional. J Bras Pneumol. 2012;38(6):803-5.

31. FILHOI, Pedro Tadao Hamamoto. Como as ligas acadêmicas podem contribuir para a formação médica?

32. Monteiro LLF, Cunha MS, Oliveira WL, Bandeira NG, Menezes JV. Ligas acadêmicas: o que há de positivo? Experiência de implantação da Liga Baiana de Cirurgia Plástica. Revista Brasileira de Cirurgia Plástica. 2001;23(3):158-61. 